\title{
Drag Performance of Twist Morphing MAV Wing
}

\author{
N.I. Ismail ${ }^{a}$, A.H. Zulkifli, R.J. Talib, H. Zaini and H.Yusoff \\ Faculty of Mechanical Engineering, Universiti Teknologi MARA, 3500 Permatang Pauh, Pulau Pinang, Malaysia
}

\begin{abstract}
Morphing wing is one of latest evolution found on MAV wing. However, due to few design problems such as limited MAV wing size and complicated morphing mechanism, the understanding of its aerodynamic behaviour was not fully explored. In fact, the basic drag distribution induced by a morphing MAV wing is still remained unknown. Thus, present work is carried out to compare the drag performance between a twist morphing wing with membrane and rigid MAV wing design. A quasi-static aeroelastic analysis by using the Ansys-Fluid Structure Interaction (FSI) method is utilized in current works to predict the drag performance a twist morphing MAV wing design. Based on the drag pattern study, the results exhibits that the morphing wing has a partial similarities in overall drag pattern with the baseline (membrane and rigid) wing. However, based $C_{D}$ analysis, it shows that $T M$ wing induced higher $C_{D}$ magnitude (between $25 \%$ to $82 \%$ higher) than to the baseline wing. In fact, $\mathrm{TM}$ wing also induced the largest $C_{D}$ increment (about $20 \%$ to $27 \%$ ) among the wings. The visualization on vortex structure revealed that TM wing also produce larger tip vortex structure (compared to baseline wings) which presume to promote higher induce drag component and subsequently induce its higher $\mathrm{C}_{\mathrm{D}}$ performance.
\end{abstract}

\section{Introduction}

Nowadays, Micro Air Vehicle (MAV) is seen as an ideal replacement for unmanned aerial vehicles (UAVs) particularly in intelligence and surveillance mission at confined space areas. With dimension lesser than $15 \mathrm{~cm}, \mathrm{MAV}$ is seen as small scale aircraft that can also fly at low-speed with Reynolds number regime between $10^{4}-10^{5}$. In order to improve the MAV performance, the biological inspired MAV design such as morphing wing is seen as a new potential to be explored[1]. Most of these biological designs are inspired from flying characteristics of airborne mammals, birds[2] and insects[3]. However, the implementation of the morphing mobility (e.g. twist morphing wing) on such limited MAV wing size wing is very challenging. The morphing MAV wing designer have to consider the limitation in wing size, limited energy, complex morphing mechanism, and high aerodynamicwing structural interaction[4]-[6]. Therefore, earlier research on such MAV wing had concentrated more on the its manoeuvrability rather than understanding its aerodynamic performance[7], [8]. Thus, the influence of morphing mobility on drag performances was not well explored. Based on this issue, current study is conducted to enhance the understanding on the drag performance of morphing MAV wing.

Here, a study is carried out by comparing the drag performance of a twist morphing MAV wing with a series of baseline MAV wing known as membrane and rigid MAV wing design. Twist

\footnotetext{
${ }^{\mathrm{a}}$ Corresponding author : iswadi558@ppinang.uitm.edu.my
} 
morphing (TM) mobility is chosen in current study due its simple deformation and actuation system. The study is mainly conducted based on quasi-static analysis by using the Fluid Structure Interaction (FSI) method. The result is elucidated through the comparison study on the drag performance between a twist morphing (TM), membrane and rigid wings..

\section{FSI Computational Method}

FSI simulation based on Ansys software is fully utilized in this work to analyze the drag performance of twist morphing, membrane and rigid wing. The combination of CFX and Static Structural module is used to solve the flow field and wing deformation, respectively. A steady state condition and incompressible turbulent flow boundary conditions is implemented to solve the airflow field. The airflow solution was obtained based on the RANS equations with Shear Stress Turbulent (SST) turbulent model. Meanwhile, Static Structural module is used to solve the linear-static structural problems on found on morphing and membrane wings. The summary of FSI simulation framework is shown in Figure 1.

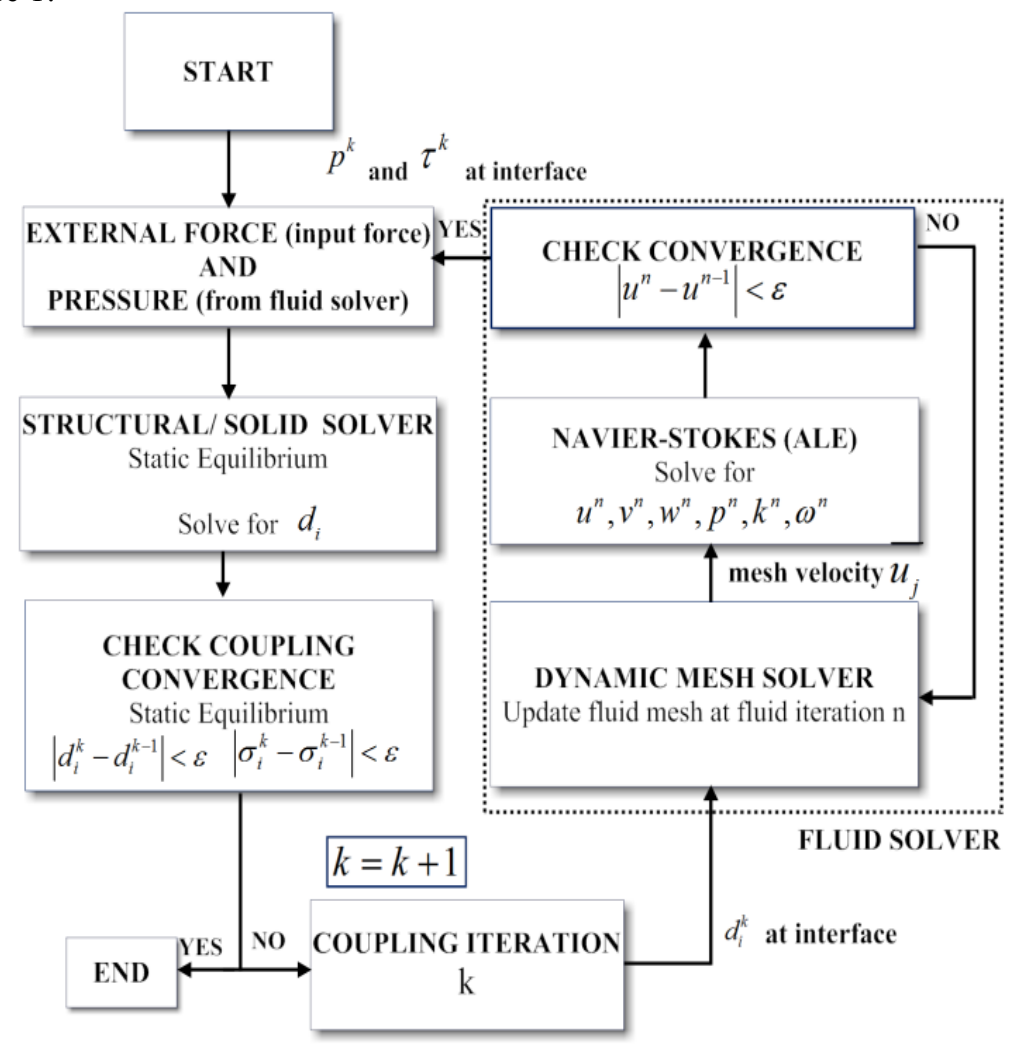

Figure 1. The summary of FSI simulation framework.

\section{MAV wing model and boundary conditions}

The twist morphing, membrane and rigid MAV wing are modeled based on previous research done at the University of Teknologi MARA[9], [10]. Due to symmetrical wing condition, all wing model are simplified into half wing. In general, all wing have almost indentical Zimmerman wing shape with wingspan, wing aspect ratio, root chord length and built-in wing twist for each wing is approximately $150 \mathrm{~mm}, 1.25,150 \mathrm{~mm}$ and $0.6^{\circ}$, respectively. However, the TM wing has included the morphing force component (for twist mobility) and membrane skin (also membrane wing). Generally, the TM 
wing design is identical to the membrane wing design but the wing has additional morphing force component $(3 \mathrm{~N})$ enforced at $90 \mathrm{~mm}$ from the leading edge and parallel to wing spanwise axis. The function of morphing force is to induce the twisted mobility on the TM wing. The thickness for wing skeleton and membrane skins is constantly at $1.0 \mathrm{~mm}$. The material for wing structure and membrane skin is set to be Perspex[10] and silicone rubber[10], respectively. The detail structure of each wing can be found in [9] and represented in Fig. 2. The mechanism to induce the morphing mobility on the TM wing is shown in Figure 3.

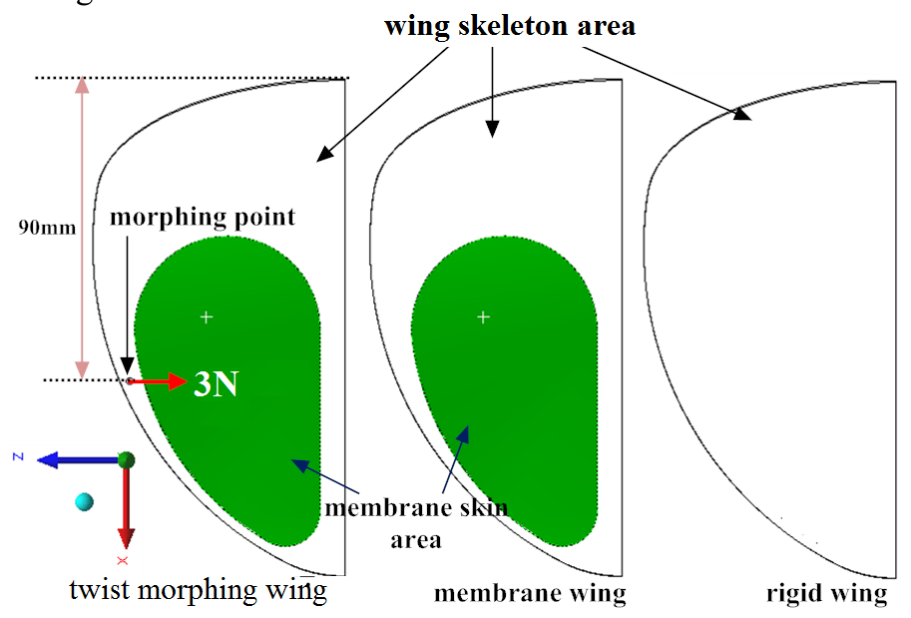

Figure 2. The TM (left), membrane (middle) and rigid wing configuration.

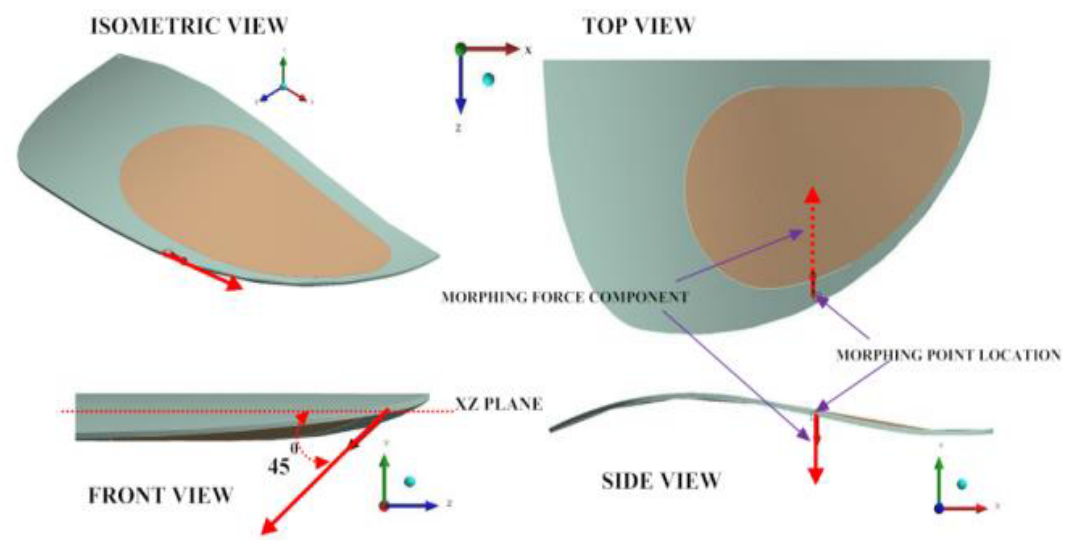

Figure 3. Mechanism principle applied on TM wing.

The size of flow domain surrounding the MAV wing is dimensioned in root chord as shown in Figure 4. The optimized number of elements for current flow domain is $1,000,000$ elements with $\mathrm{y}^{+}$is obtained below than 1.The inlet, outlet and symmetrical wall location are shown in Figure 4 . The airflow speed is imposed at the inlet and set at $9.7 \mathrm{~m} / \mathrm{s}$ which is equivalent to $\mathrm{Re}=100,000$ at chord (define as the maximum speed for MAV). The angle of attack (AOA) increment starts form $-7^{\circ}$ to $30^{\circ}$ with $2^{\circ}$ interval. The turbulence intensity of $5 \%$ with automatic wall function is also imposed to solve the flow viscous effect. 


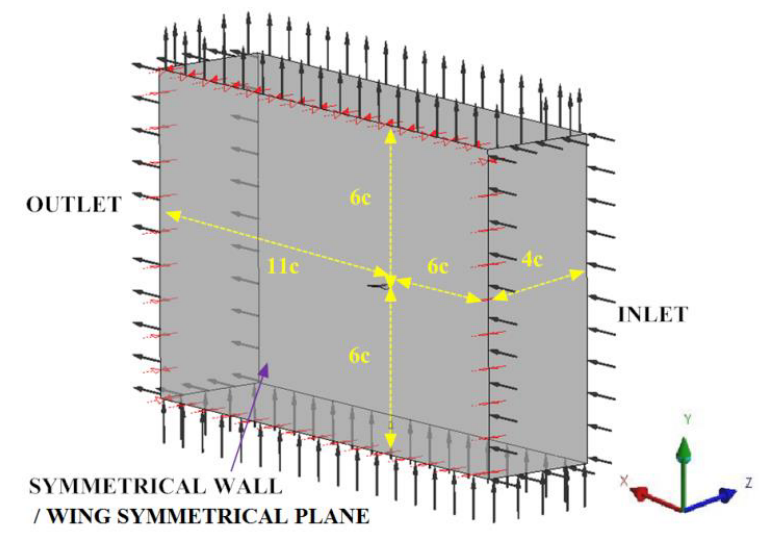

Figure 4. flow domain dimensioned in the wing root chord.

\section{Drag coefficient results}

The drag coefficient $\left(C_{D}\right)$ results for all wings are shown in Fig. 4. The result shows that all wings had exhibited almost a constant $C_{D}$ magnitude at the early AOA stages between $-15^{\circ}$ to $2^{\circ}$. However $C_{D}$ magnitude for all wings had drastically increase after the AOA increase higher than $2^{\circ}$. The increment of $C_{D}$ magnitude was slight dented at stall angle (between $A O A=22^{\circ}$ to $26^{\circ}$ ) before the $C_{D}$ value increase further almost linearly up to $A O A=35^{\circ}$. In general, the $C_{D}$ magnitude produced by the baseline wing is almost identical. However, the membrane wing managed to induced slightly ( $2 \%$ to $3 \%$ ) higher $\mathrm{C}_{\mathrm{D}}$ magnitude compared to the rigid wing.

Based on the detail analysis on the magnitude of $\mathrm{C}_{\mathrm{D}}$, the result shows that $\mathrm{TM}$ wing able to produce lower $\mathrm{C}_{\mathrm{D}}$ magnitude (compared to baseline wing) at the early AOA stages (between $-15^{\circ}$ to $8^{\circ}$ ). At this AOA stage, TM wing exhibited about $7 \%$ to $18 \%$ lower $\mathrm{C}_{\mathrm{D}}$ magnitude compared to baseline wing. The minimum $C_{D}$ magnitude found on TM wing is 0.0363 exhibited at $A O A=-6^{\circ}$. However, the minimum $C_{D}$ magnitude for $T M$ wing is induced at earlier angle compared to the baseline wing $\left(\mathrm{AOA}=-2^{\circ}\right)$. As the AOA increase $\left(\mathrm{AOA}>-2^{\circ}\right)$, the $\mathrm{C}_{\mathrm{D}}$ value for $\mathrm{TM}$ wing had also significantly increase. Analytically, TM wing managed to produce about $25 \%$ to $82 \%$ higher $C_{D}$ magnitude than the baseline wing at AOA between $-2^{\circ}$ to $22^{\circ}$. TM wing stalled at $A O A=22^{\circ}$ with $C_{D}$ $=0.557$ which is about $47 \%$ higher than the baseline wing produced. The stall angle for the baseline wing delayed at $A O A=26^{\circ}$. Based on depth $C_{D}$ analysis, TM wing exhibited a drastic increment in $C_{D}$ magnitude especially at AOA between $-2^{\circ}$ to $12^{\circ}$. At this AOA stage, the $\mathrm{C}_{\mathrm{D}}$ magnitude for TM wing increase about $20 \%$ to $27 \%$ for every $2^{\circ}$ AOA increment. Despite of high $\mathrm{C}_{\mathrm{D}}$ increment found in $\mathrm{TM}$ wing, the baseline just induced slightly lower $\mathrm{C}_{\mathrm{D}}$ increment than the TM wing at the same AOA range $\left(-2^{\circ}\right.$ to $\left.12^{\circ}\right)$. The baseline wing exhibited about $14 \%$ to $24 \%$ of $C_{D}$ increment for every $2^{\circ}$ AOA rise. However, as the AOA increase higher than $12^{\circ}$ (up to $22^{\circ}$ ), the increment of $C_{D}$ magnitude for both $\mathrm{TM}$ and baseline wings had reduced below than $10 \%$.

Based on this result, it clearly shows that TM wing had induced higher $\mathrm{C}_{\mathrm{D}}$ distribution compared to the baseline wing particularly at every AOA cases higher than $-2^{\circ}$. Based on previous study shown in references [9], [10], this malevolent performance is partially contributed by the enlargement of tip vortex structure induced by TM wing. Larger tip vortex structure contributed into higher induce drag component in which consequently contributed into higher drag performance[11]. Thus, a preliminary investigation on the tip vortex structure found on TM and baseline wings is carried out to justify the drag distribution findings.

Figure 6 shows the vortex structure found on TM and baseline wings at $\mathrm{AOA}=5^{\circ}$ and $10^{\circ}$. The vortex structure result is visualized based on $\mathrm{Q}$ criterion magnitude equal to 0.04 and the tip vortex structures are recognized based on its location that induced at the wing tip as shown in Figure 5. The airflow moves from top to the bottom of the page. Based on this figure, it clearly shows that each 
wing produce tip vortex structure that flows downstream behind the wing tips. Based on relative comparison (estimated by the relative length and diameter), the results clearly shows that TM wing induce larger tip vortex structure compared to the baseline wings. In fact, the size of vortex structure for TM (and also baseline) wing continue to increase as the AOA increase from $5^{\circ}$ to $10^{\circ}$. However, the size of tip vortex structure for rigid and membrane wings are almost identical based on estimated length and diameter. Thus, the results explain the reason behind the enormous $C_{D}$ distribution found on TM wing. Larger tip vortex structure found on TM wing induce its induce drag component which subsequently contribute in amplifying its overall $C_{D}$ performance. On the other hand, $C_{D}$ performance for rigid and membrane wings are almost identical due to the similarities (in terms of size) of tip vortex structure found on both wings.

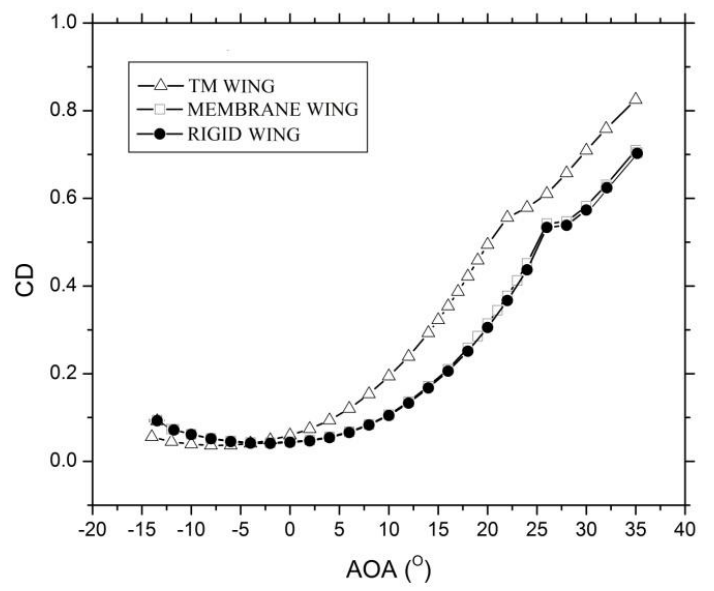

Figure 5. Drag performance for twist morphing, membrane and rigid wings.

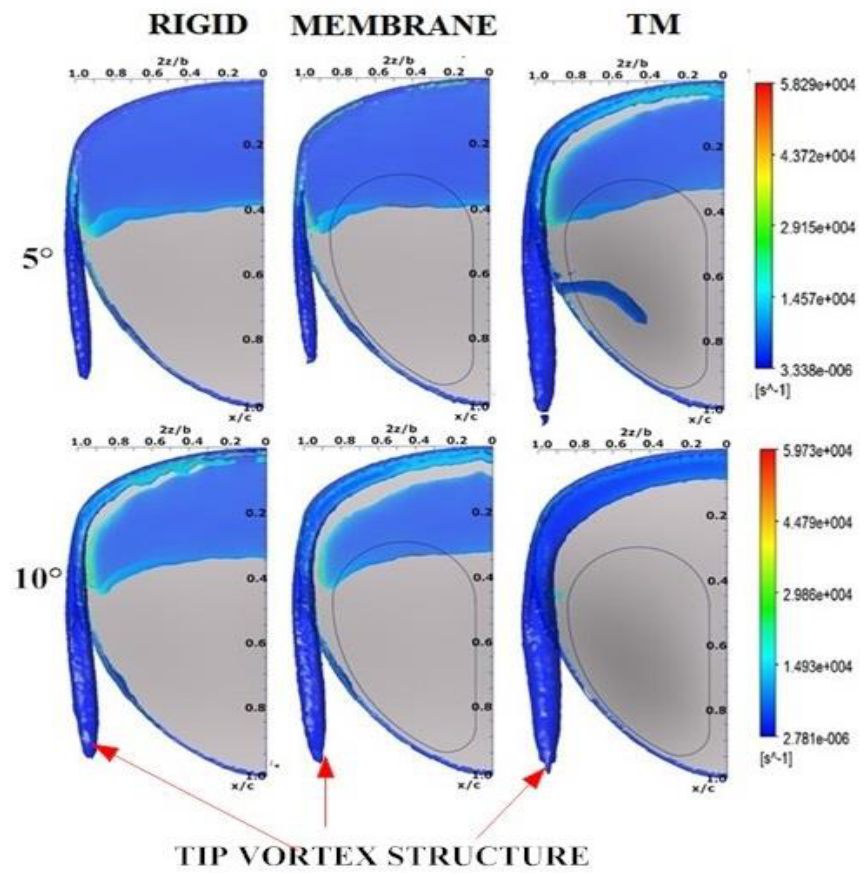

Figure 6. The vortex structure found on $\mathrm{TM}$ and baseline wings at $\mathrm{AOA}=5^{\circ}$ and $10^{\circ}$. 


\section{Conclusion}

Understanding the basic drag performance of morphing MAV wing is very crucial in order to enhance its application in MAV area. Thus, current study is carried out to compare the drag performance of a twist morphing MAV wing with membrane and rigid wings. Based on initial drag pattern study, the results indicates that the wings have a partial similarities in overall drag pattern. However, based on the detail $\mathrm{C}_{\mathrm{D}}$ analysis, the results clearly shows that $\mathrm{TM}$ wing induced higher $\mathrm{C}_{\mathrm{D}}$ magnitude (between $25 \%$ to $82 \%$ ) compared to the baseline wing in most AOA cases. In fact, TM wing induced the largest $\mathrm{C}_{\mathrm{D}}$ increment among the wings (about $20 \%$ to $27 \%$ ) at AOA between $-2^{\circ}$ to $12^{\circ}$. The visualization on vortex structure also revealed that TM wing also produce larger tip vortex structure compared to baseline wings. Larger tip vortex structure found on TM wing presume to promote higher induce drag component and subsequently induce its higher $\mathrm{C}_{\mathrm{D}}$ performance.

\section{Acknowledgments}

The authors acknowledge technical and financial support from Universiti Teknologi MARA and the Government of Malaysia via the sponsorship by the Malaysia Ministry of Higher Education's Fundamental Research Grant Scheme (FRGS) (600-RMI/FRGS 5/3 (152/2014)).

\section{References}

1. M. Abdulrahim, H. Garcia, and R. Lind, J. Aircr., 42, 131 (2005)

2. A. H. Supekar, Design, Analysis and Development of A Morphable Wing Structure For Unmanned Aerial Vehicle Performance Augmentation, , (University of Texas, 2007)

3. S. A. Combes and T. L. Daniel, J. Exp. Biol., 206, 2989, (2003)

4. Z. Chen, Micro Air Vehicle Design for Aerodynamic Performance and Flight Stability, (University of Sheffield, 2013)

5. R. Banerjee and P. Seshaiyer, Int. J. Aerosp. Light. Struct., 3, 1, (2013)

6. Y. J. Abudaram, S. Rohde, J. P. Hubner, and P. Ifju, Composite Materials and Joining Technologies for Composites, (Springer New York, 2013)

7. R. Pecora, F. Amoroso, and L. Lecce, J. Aircr., 49, 1666 (2012)

8. S. Vasista, L. Tong, and K. C. Wong, J. Aircr., 49, 11 (2012)

9. N. I. Ismail, A. H. Zulkifl, M. Z. Abdullah, M. H. Basri, N. S. Abdullah, A. H. Zulkifli, M. Hisyam Basri, and N. Shah Abdullah, Chinese J. Aeronaut., 26, 1093 (2013)

10. N. I. Ismail, a. H. Zulkifli, M. Z. Abdullah, M. H. Basri, and N. S. Abdullah, Chinese J. Aeronaut., 27, 475 (2014)

11. B. K. Stanford, P. Ifju, R. Albertani, and W. Shyy, Prog. Aerosp. Sci.,44, 258 (2008) 